\title{
Fault diagnosis of wind turbine based on
}

\section{rough set and BP network}

\author{
Xinli Li ${ }^{1,}$, , Wanye $\mathrm{Yao}^{2, \mathrm{~b}}$,Qingjie Zhou ${ }^{3, \mathrm{c}}$ \\ Jianming Wang ${ }^{4, d}$,Jingzhi Liu ${ }^{5, e}$ \\ ${ }^{1}$ School of Control and Computer Engineering, North China Electric Power University, \\ Baoding,Hebei, 071003, China \\ ${ }^{2}$ Beijing Join Bright digital power technology Co,Ltd, Beijing,100085,China

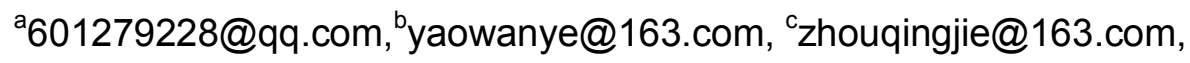

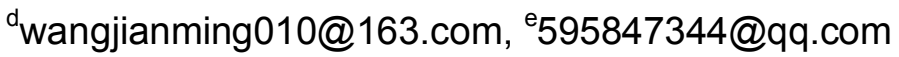

Keywords: Rough set, BP neural network, Fault niagnosis,Pitch system

Abstract. Because of wind turbines institutional complexity, many operating parameters, fuzziness,randomness and uncertainty between the cause of the fault and the fault symptom, so direct application of BP neural network for fault diagnosis can lead to the issues of slow convergence of neural network and long training time, therefore we need take some method reducing the speed and improve accuracy of diagnosis. for this case, the fault diagnosis method for wind turbine based on rough set and BP neural network is presented in this paper. Firstly, rough set theory is a effective tool to deal with vagueness and uncertainty of knowledge, it can simplify the decision-making rules, and can get rid of redundant information with the classification ability unchanged,then put the simplified information into the BP neural network for training,this method can reducing the input dimension of BP neural network to improve diagnostic accuracy and reduce training time. Simulation results show that: the use of this diagnostic method in the wind turbine pitch system, the relationship between the established model accuracy is relatively high, it can make a rapid and accurate diagnosis for the operating status and the fault of turbine pitch system.

\section{Introduction}

With the development of science and production technology, wind turbine structure equipment more and more complexs, once the wind turbines created failure it will cause huge economic losses, it is necessary to created a fault diagnosis system can quickly find the cause of the fault,in order to quickly treatment. In recent years, with the rapid development of artificial intelligence technology,resulting in a fault diagnosis method based on knowledge, this method does not require precise mathematical model due to the object, but also has some "smart" characteristics, so it has been widely used [1]. Knowledge-based fault diagnosis method can be divided into: expert system fault diagnosis: fuzzy fault diagnosis; fault tree for fault diagnosis; neural network fault diagnosis [2-3]. Neural network fault diagnosis method due to have parallel computing, self-organization and nonlinear mapping ability, so in fault diagnosis has been widely studied and applied [4]. But the BP neural network in the practical application of the following shortcomings: One can not identify the redundant information; the second is unable to identify new fault samples; the third is for a complex 
system, the training required for a long time, not conducive to real-time diagnostics [5]. Rough set theory does not require any a priori knowledge to the uncertain, inconsistent and incomplete multi-source information for effective analysis and processing, but also to discover hidden knowledge which reveals the internal laws, the use of attribute reduction theory diagnostic process a large number of redundant features reduction, so the introduction of rough set theory in neural network fault diagnosis can reduce the input dimension of the neural network, it improve the accuracy of diagnosis, reduce training time, but the rough set method sensitive to the noise and inaccurate classification problems exist the large noiseenvironments,, while the neural network will have the ability to suppression the noise[6]. Therefore, combined the rough set theory and neural networks to improve the accuracy of diagnosis is necessary.

The basic idea of rough set-BP neural network fault diagnosis is: extracting failures date form the SCADA system, after a preliminary analysis get the fault feature sample of pitch system; the pitch system features characteristic parameters as a condition property, the corresponding fault category as the decision attribute,construct an initial sample decision table; then discrete the samples by SOM algorithm and intervals encodes to get a decision table, and then get most simple decision table after the attribute reduction algorithm by difference matrix; finally, the minimum condition attribute as BP neural network input neurons after reduction of rough, the type of fault as a neural network output unit, configured BP neural network,then learning and training the network make it has association and memory function;after the training completion, input the failure samples to the trained neural network, finally get the type of fault.Fault diagnosis process shown in Fig.1 :

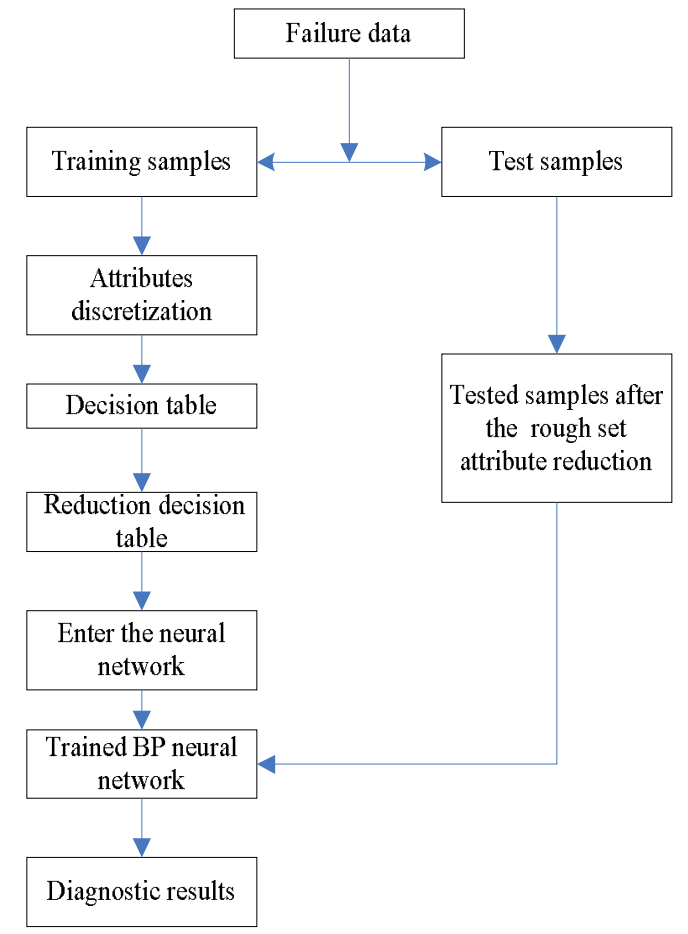

Fig.1 Fault diagnosis process

\section{Rough set theory}

The basic idea of rough set attribute reduction: $\mathrm{R}$ indiscernible relation to division the knowledge of domain $U$, composition a knowledge representation system $K=(U, R)$, use of approximation set and lower approximation approaching the object;check the decision table for delete the duplicate rules; then use the following attribute reduction algorithm for attribute reduction, get the simplified decision table; final consistency inspection again of the decision table, repeatability and deleting 
unimportant text lines to achieve the reduction of space vector[7]. Moreover, because the rough set theory only analysis and processing to the discrete attribute value, while the characteristic parameters of pitch systems most of continuous quantity. Hence before the reduction need discrete processing, this paper uses SOM algorithm for data discrete processing.

The most commonly used attribute reduction method is based on heuristic of difference matrix for attribute reduction [7-8]:

(1) Construct difference matrix (M)base on information systems, $m_{i j}$ is defined as follows:

$m_{i j}=\left\{\begin{array}{l}\left\{f \mid f \in R \wedge f\left(x_{i}, f\right) \neq f\left(x_{j}, f\right)\right\},\left(x_{i}, x_{j}\right) \in \operatorname{ind}(P) \\ \phi,\left(x_{i}, x_{j}\right) \in \operatorname{ind}(P)\end{array}\right.$

(2)All the elements properties of $\mathrm{M}$ constituted relative nuclear $\mathrm{B}=\mathrm{Core}_{\mathrm{D}}(\mathrm{F})$

(3)For $\forall m_{i j} \in M, i, j=1,2 \ldots|| U \mid$. ,if $A \cap m_{i j} \neq \phi$, then $m_{i j}=\phi$

(4)For $\forall m_{i j} \in M, i, j=1,2 \ldots|U|$, if $m_{i j}=\phi$, then (6) , or turn (5)

(5)Select any one of the conditions f of property does not belong to A, For $A=A \cup\{f\}$, turn (3)

(6)End of the algorithm, output the knowledge reduction set.

\section{BP neural network}

BP neural network is a multilayer feedforward neural network, the basic idea is : signal feedforwar: the input signal from the input layer, hidden layer through layer by layer processing until the output layer, each layer neuron state only affects the next layer neuron state; error backpropagation: if one neuron output layer is not obtain the expected output, then the error value feedback to enter back propagation stage, according to the prediction error to adjust the network weights and threshold, so that the predicted output of BP neural network constantly approaching the desired output[9].

BP neural network training process as follows [10]:

(1)Network initialization. According to the number of input and output samples to determine the input layer nodes $\mathrm{n}$, hidden layer nodes $\mathrm{h}$, the output layer nodes $\mathrm{c}$; the connection weights $\omega_{i j}$ between the input layer and the hidden layer, the connection weights $\omega_{j k}$ between the hidden layer and output layer, the hidden layer threshold is a, the output layer threshold is b, the random number is initialized to a minimum. Given the learning rate $\eta$ and neuronal excitation functions.

(2)Calculate the hidden layer output $\mathrm{H}: H=f\left(\sum_{i=1}^{n} \omega_{i j} x_{i}-a_{j}\right), j=1,2 \ldots h, f$ is the hidden layer activation function.

(3)Calculate the output layer output C: $C_{k}=\sum_{j=1}^{h} H_{j} \omega_{j k}-b_{k}, k=1,2 \ldots c$

(4)Error calculation: $e_{k}=Y_{k}-C_{k}, \quad Y$ is the expected output 
(5)Adjust the weights: $\omega_{i j}=\omega_{i j}+\eta H_{j}\left(1-H_{j}\right) x(i) \sum_{k=1}^{m} \omega_{j k} e_{k} \quad \omega_{j k}=\omega_{j k}+\eta H_{j} e_{k}$

(6)Adjust the threshold: $a_{j}=a_{j}+\eta H_{j}\left(1-H_{j}\right) \sum_{k=1}^{m} \omega_{j k} e_{k} \quad b_{k}=b_{k}+e_{k}$

(7)Determines whether the end of the algorithm, if the end condition is not reached, the process returns to step (2).

\section{Rough Set-BP network fault diagnosis application in pitch system}

\section{Fault diagnosis decision table.}

Collecting representative of failure data 300 groups around the pitch system failure from the SCADA system, all normalized to the interval [0,1], with which 200 sets of data as training samples, and the remaining 100 sets of data as test data. Select three common faults from pitch system as follows: fault 1: pitch motor high temperature, which trigger condition is the pitch motor temperature higher than 150 degrees continuous 3s; fault 2: pitch encoder fault, which trigger condition is the wind detected pitch rotary encoder output values overflow signal; fault 3: pitch capacitor voltage imbalance fault, its trigger condition is a low voltage capacitor pitch, duration $3 \mathrm{~s}$ larger than the capacitance of the high pitch plus half the voltage $4 \mathrm{~V}$, fans reported this fault; these three fault as the decision attribute, in decision table with g1, g2, g3 represent. According to the fault trigger conditions selected fault characteristic parameters about 15: wind speed, generator speed, power, \# 1pitch angle, \# 2 pitch angle, \# 3pitch angle , \# 1 pitch rate change \# 2 paddle rate, \# 3pitch rate, \# 1 pitch cabinet capacitor high voltage, 2 \# pitch cabinet capacitor high voltage, 3 \# pitch cabinet capacitor high voltage, 1 \# pitch motor temperature, 2 \#motor temperature pitch,3 \# pitch motor temperature; this 15 characteristic parameters as a condition of property, indicated by $c_{1} \sim c_{15}$. According to the above conditions, we can construct an initial decision table, and then use the SOM algorithm attributes discrete processing conditions table, take $[0,0.3]$ interval coded as $0,[0.3,0.6]$ interval coded as 1 , [ $\left.\begin{array}{ll}0.6 & 0.9\end{array}\right]$ interval coded as 2, [0.9-1] interval coded as 3 , after the discrete 10 groups are shown in Table 1:

Table 1 Decision table

\begin{tabular}{|c|c|c|c|c|c|c|c|c|c|c|c|c|c|c|c|c|}
\hline \multirow{2}{*}{$\begin{array}{l}\text { Fault } \\
\text { sampl }\end{array}$} & \multicolumn{15}{|c|}{ Condition attributes } & \multirow{3}{*}{$\begin{array}{l}\text { faul } \\
\mathrm{t} \\
\text { type }\end{array}$} \\
\hline & $\mathrm{c}$ & $\mathrm{c}_{2}$ & $\mathrm{c}$ & $\mathrm{c}_{4}$ & $\mathrm{c}_{5}$ & $\mathrm{c}_{6}$ & $\mathrm{c}_{7}$ & $\mathrm{c}_{8}$ & $\mathrm{c}_{9}$ & $\mathrm{c}_{10}$ & $\mathrm{c}_{11}$ & $\mathrm{c}_{12}$ & $\mathrm{c}_{13}$ & $\mathrm{c}_{1}$ & $\mathrm{c}_{15}$ & \\
\hline es & 1 & & 3 & & & & & & & & & & & 4 & & \\
\hline 1 & 1 & 3 & 2 & 0 & 0 & 0 & 1 & 1 & 2 & 3 & 3 & 3 & 2 & 3 & 3 & g1 \\
\hline 2 & 1 & 3 & 2 & 0 & 0 & 0 & 2 & 2 & 2 & 3 & 3 & 3 & 2 & 3 & 3 & g1 \\
\hline 3 & 0 & 1 & 2 & 0 & 0 & 0 & 0 & 0 & 1 & 3 & 3 & 3 & 0 & 0 & 0 & g2 \\
\hline 4 & 1 & 3 & 2 & 0 & 0 & 0 & 0 & 0 & 1 & 3 & 3 & 3 & 2 & 3 & 3 & g1 \\
\hline 5 & 0 & 1 & 2 & 0 & 0 & 0 & 0 & 0 & 2 & 3 & 3 & 3 & 0 & 0 & 0 & g2 \\
\hline 6 & 1 & 3 & 3 & 0 & 0 & 0 & 1 & 1 & 2 & 3 & 3 & 3 & 0 & 0 & 0 & g3 \\
\hline 7 & 0 & 1 & 2 & 0 & 0 & 0 & 0 & 0 & 0 & 3 & 3 & 3 & 0 & 0 & 0 & g2 \\
\hline 8 & 2 & 3 & 3 & 1 & 1 & 1 & 1 & 1 & 2 & 1 & 1 & 1 & 0 & 0 & 0 & g3 \\
\hline 9 & 2 & 3 & 3 & 0 & 0 & 0 & 1 & 1 & 2 & 3 & 3 & 3 & 0 & 0 & 0 & g3 \\
\hline 10 & 0 & 1 & 2 & 0 & 0 & 0 & 0 & 0 & 3 & 3 & 3 & 3 & 0 & 0 & 0 & g2 \\
\hline
\end{tabular}

Then use the attribute reduction algorithm based on discernibility matrix processing simple decision 
table, the minimum simplified decision table is $\left\{c_{1}, c_{2}, c_{3}, c_{5}, c_{9}, c_{10}, c_{13}\right\}$, As shown in table 2:

Table 2 Minimum simplified decision table

\begin{tabular}{cllllllll}
\hline Fault & \multicolumn{7}{c}{ Condition attributes } & \multirow{2}{*}{ fault type } \\
\cline { 2 - 7 } samples & $\mathrm{c}_{1}$ & $\mathrm{c}_{2}$ & $\mathrm{c}_{3}$ & $\mathrm{c}_{5}$ & $\mathrm{c}_{9}$ & $\mathrm{c}_{10}$ & $\mathrm{c}_{13}$ & \\
\cline { 2 - 7 } 1 & 1 & 3 & 2 & 0 & 2 & 3 & 2 & $\mathrm{~g} 1$ \\
2 & 1 & 3 & 2 & 0 & 2 & 3 & 2 & $\mathrm{~g} 1$ \\
3 & 0 & 1 & 2 & 0 & 1 & 3 & 0 & $\mathrm{~g} 2$ \\
4 & 1 & 3 & 2 & 0 & 1 & 3 & 2 & $\mathrm{~g} 1$ \\
5 & 0 & 1 & 2 & 0 & 2 & 3 & 0 & $\mathrm{~g} 2$ \\
6 & 1 & 3 & 3 & 0 & 2 & 3 & 0 & $\mathrm{~g} 3$ \\
7 & 0 & 1 & 2 & 0 & 0 & 3 & 0 & $\mathrm{~g} 2$ \\
8 & 2 & 3 & 3 & 1 & 2 & 1 & 0 & $\mathrm{~g} 3$ \\
9 & 2 & 3 & 3 & 0 & 2 & 3 & 0 & $\mathrm{~g} 3$ \\
10 & 0 & 1 & 2 & 0 & 3 & 3 & 0 & $\mathrm{~g} 2$ \\
\hline
\end{tabular}

\section{Build fault diagnosis model}

(1)Setting network parameters: condition attribute simples is 7 , so the input layer of the neural network innum $=7$, select the pitch system of three failures, so neural network output layer outnum $=$ 3 , error precision is set to 0.0001 ; because the hidden layer nodes have no precise theoretical basis, so after a preliminary test, the hidden layer node midnum $=10$. During training, there is no laws of the change experimental error, the success of network training to go through a lot of work to verify. The entire training process requires repeated testing, repeatedly try different combinations of excitation functions, and ultimately select the hidden layer activation function for logsig; after parameter setting, initialize the network weights $\omega_{1}, \omega_{2}$ and thresholds $b_{1}, b_{2}$;

(2)Weights and thresholds adjustments: reverse transfer function of neural networks is continuously adjusted the process of error weights, the program to achieve is following: $\mathrm{dw} 1(\mathrm{k}, \mathrm{j})=\mathrm{FI}(\mathrm{j}) * \mathrm{x}(\mathrm{k}) *(\mathrm{e}(1) * \mathrm{w} 2(\mathrm{j}, 1)+\mathrm{e}(2) * \mathrm{w} 2(\mathrm{j}, 2)+\mathrm{e}(3) * \mathrm{w} 2(\mathrm{j}, 3))$ $\mathrm{db} 1(\mathrm{j})=\mathrm{FI}(\mathrm{j}) *(\mathrm{e}(1) * \mathrm{w} 2(\mathrm{j}, 1)+e(2) * w 2(\mathrm{j}, 2)+e(3) * w 2(\mathrm{j}, 3))$;

(3) Figure 2 is a rough set- BP neural network method predictions results

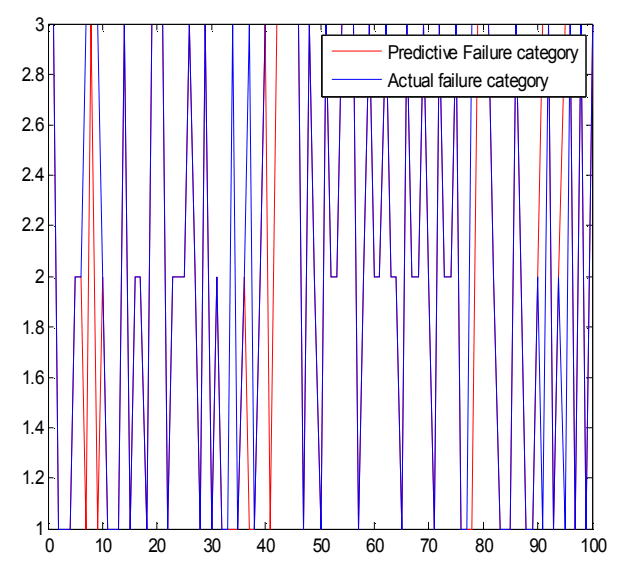

Fig. 2 rough set- BP neural network predictions results

Analysis:form the fig.2 is easy to see, the test sample predicted output and the actual output is very close, indicating that the neural network model build better. 
(4)error conditions of directly use BP neural network fault classification as shown figure 3, and the rough set-BP neural network method of fault classification shown in figure 4

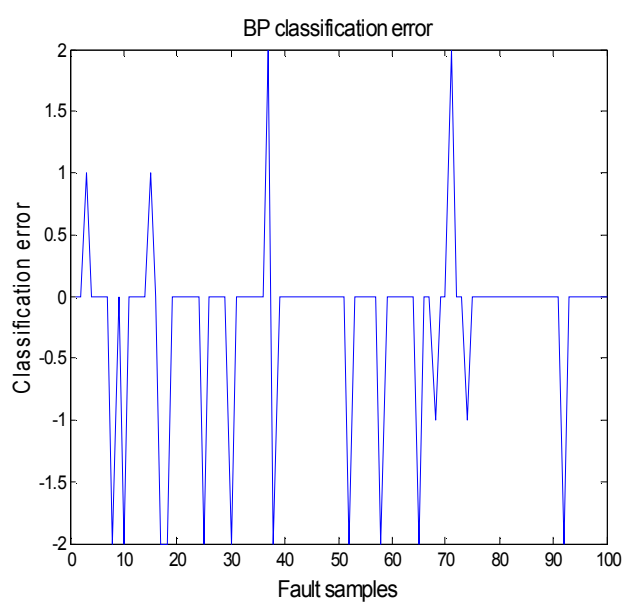

Fig.3 BP classification error

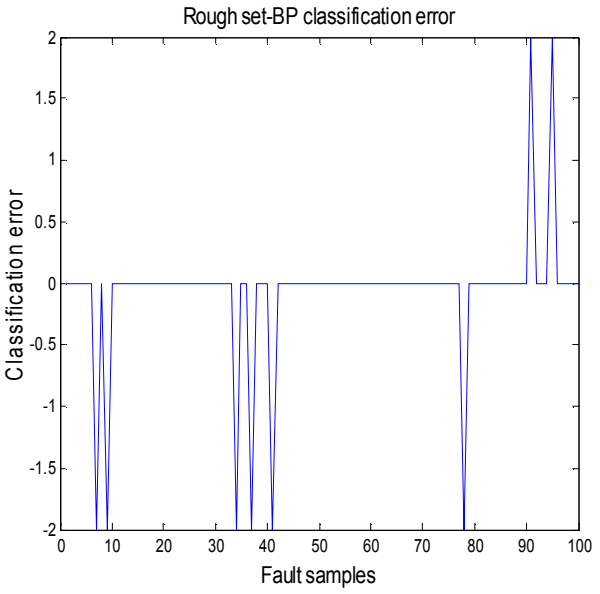

Fig.4 rough set-BP classification error

Analysis: from Fig. 3 and Fig. 4 we can clearly see: use of rough sets-BP neural network method the error is smaller; we can summary that the model application is effective.

(5)In order to more clearly reflect the difference between the two method, the table 5 will shown the BP neural network classification accuracy and the rough set- BP neural network accuracy compares results:

Table 5 compares results

\begin{tabular}{ccc} 
Fault type & \multicolumn{2}{c}{ Correct rate } \\
\cline { 2 - 3 } g1 & BPneural network & rough set- BP neural network \\
\cline { 2 - 3 } g2 & 0.7911 & 0.9297 \\
g3 & 0.9017 & 1.0000 \\
& 0.7917 & 0.8798
\end{tabular}

Analysis:the table 5 shows that the rough set-BP neural network method for pitch fault diagnosis system, the type of fault is higher accuracy rate.

(6)Form the SCADA system get 10 groups pitch motor temperature high fault data, input to the fault diagnosis model to obtain results as shown in Table 6:

Table 6 Diagnostic results

\begin{tabular}{|c|c|c|c|}
\hline $\begin{array}{c}\text { Diagnostic } \\
\text { results }\end{array}$ & $\mathrm{g} 1$ & $\mathrm{~g} 2$ & $\mathrm{~g} 3$ \\
\cline { 2 - 4 } & 0.0049 & 0.9801 & 0.0150 \\
\hline
\end{tabular}

Analysis: the table 6 shown that use of rough sets-BP neural network get the results of fault diagnosis is $\mathrm{g} 2$, which is pitch motor temperature fault, the actual results and fault diagnosis has consistent.It's result indicate that the method has a higher effectiveness.

\section{Summary}

Considering the ambiguity and uncertainty of wind turbine pitch systems between the cause of the fault and the fault symptom, so make full use of the rough theoretical advantages in dealing with incomplete information is uncertain terms, make the rough set theory and BP neural network combining fault diagnosis method is introduced in wind turbine pitch system. Diagnostic analysis through the application of a wind turbine pitch system actual failure data, indicating that the fault diagnosis method can effectively improve the diagnostic accuracy of the neural network, compensate for the lack of traditional BP neural network. The fault diagnosis method for dealing with large 
volumes of data, the structure of the complex fault diagnosis of wind turbines has some practical value.

\section{References}

[1] Xie Fangfang.Support Vector Machine for fault diagnosis[D].Hunan University, (2006)

[2] Yang Shuzi,Shi Tielin,Knowledge-based diagnostic reasoning.BeiJing:Tsinghua University Press,(1993)

[3] Shigeo Abe.Support vector machines for pattern recognition. New York:Spiinger-Verlag,(2004):15-30

[4] Wei Mingshe,Li Guoyong.FNN-based intelligent fault diagnosis for ship-diesel engine[J].Computer Simulation,(2007),24(12):159-162

[5] Tan Tianle,Song Zhihuan,Li Ping.Approach for fault detection and diagnosis based on rouhg set.Zhejiang University[J].(2003),37(1):47-50

[6] Yang Dan. The research of fault diagnosis method based on rough sets and neural network[D].Jiangnan University,(2008)

[7] Wang Ping.Research and Application of Categorization Algorithms Based on Rough Sets Attributes Reduction[D].Dalian University of Technology,(2013)

[8] PAWLAKZ.Rough sets theory and knowledge acquisition[M].Xi'an:Xi'an Jiaotong University Press,(2001)

[9] Xu Xianghe.Customer classification method based on BP ANN[D].Nanjing: Nanjing University of Aeronautics and Astronautics,(2004)

[10] Zhou Zhihua,Cao Cungen.Neural Network and Its Application[M].Beijing:Qing Hua University Press,(2004) 